there are neurones in the pineal parenchyma of adults, located either singly or in a ganglion. Although it is unclear whether they are of pineal origin or are derived from the habenula, it is now thought that these neurones and adjacent secretory pinealocytes are in synaptic contact with central fibres innervating the gland via the habenula and posterior commissures (see figure) (Korf H.-W. \& Moller, M. Pineal Res. Rev. 2, 41; 1984). This necessitates modification of the established model of solely autonomic regulation of pineal activity. Adding another twist to the problem of pineal-brain communication, Korf et al. in their new work use an antiserum against bovine retinal S-antigen (a specific marker for photoreceptive elements) to stain cells in the pineal of the Djungarian hamster. Expression of the antigen by presumed secretory pinealocytes supports the earlier conclusion, based on comparative anatomical and developmental evidence, that these cells derive from an ancestral photoreceptor. Immunoreactive cells extend throughout the pineal but scattered elements are found in the habenula. These scattered cells may be pinealocytes in an aberrant position, but the possibility that some habenula neurones express the antigen cannot be excluded. How can this problem be resolved?

The pineal of the Djungarian hamster is divided into deep and superficial parts connected by a narrow stalk (see figure). The deep pineal and habenula share the autonomic innervation of the superficial gland, but the deep pineal has no rhythm of melatonin production and is not involved in photoperiodic repsonses. Korf $e t$ al. observe that the labelled cells of the deep pineal project to the adjacent habenula and posterior commissure. The varicose morphology of the projections is similar to the form of neuronal axons commonly encountered elsewhere in the central nervous system, which implies that the projections form an anatomical basis for neural communication between electrically active secretory pinealocytes and the brain. This novel form of output appears to strengthen the functional association of the mammalian pineal with that of the poikilotherms.

But, if these processes do represent a neural output, such afferent innervation is very different from that of lower vertebrates and fetal mammals. First, the pinealofugal processes are extensions of the pinealocyte, and not of ganglion cells; second, the projections do not form a single, definable nerve tract but seem to run irregularly thoughout the epithalamic region; and finally, there is no evidence for sensory receptor function in the mammalian pineal. Consequently, information relayed along this route would presumably be interoceptive and one can only speculate on its function. If pinealo- cytes are scattered throughout the epithalamus, is it possible that the connections are responsible for the synchronization of metabolic activity? Alternatively, the processes may indeed be neurally active, conveying afferent impulses to synaptic contacts with central neurones.

Korf et al. speculate that melatonin is a transmitter in the centrally directed processes. But pinealocytes are known to contain large amounts of unidentified secretory material, particularly peptides, and there is no a priori reason why melatonin should be the only product that can fulfill this function. Indeed, the biosynthesis and release of melatonin from the superficial pineal suggests that specialized storage and release mechanisms, a characteristic feature of conventional neurotransmitter systems, do not exist in this case. Melatonin released from the processes may have a paracrine function in the region of the epithalamus parallel to its proposed control of pigment migration in photoreceptive structures. Such an effect in the brain would be unusual and available data suggest that melatonin acts in the hypothalamus, some distance away.
To make functional sense of the putative central projections of the pineal that I have described here, there must be a precise description of their sites of termination and synaptic associations with central neurones. In addition, to understand pineal physiology in mammals, functions other than photoperiodic time measurement and the melatonin signal must be identified. Should pineal peptides other than melatonin be shown to have regulatory functions, these transmitters could mediate a central action complementary to the humoral effect, following the precedent of the small neuropeptides of the hypothalamus. Of course, parallels between the epithalamus and hypothalamus were suggested years ago (Roussy \& Mosinger C.r. Soc. Biol., Paris 127,655 ; 1938). Perhaps these early anatomists were able to use perceptive pathways no longer exercised in their modern counterparts.

Michael Hastings is a demonstrator in the Department of Anatomy, University of Cambridge, Downing Street, Cambridge CB2 $3 D Y, U K$

\title{
Structures in the gas tail of comet Halley
}

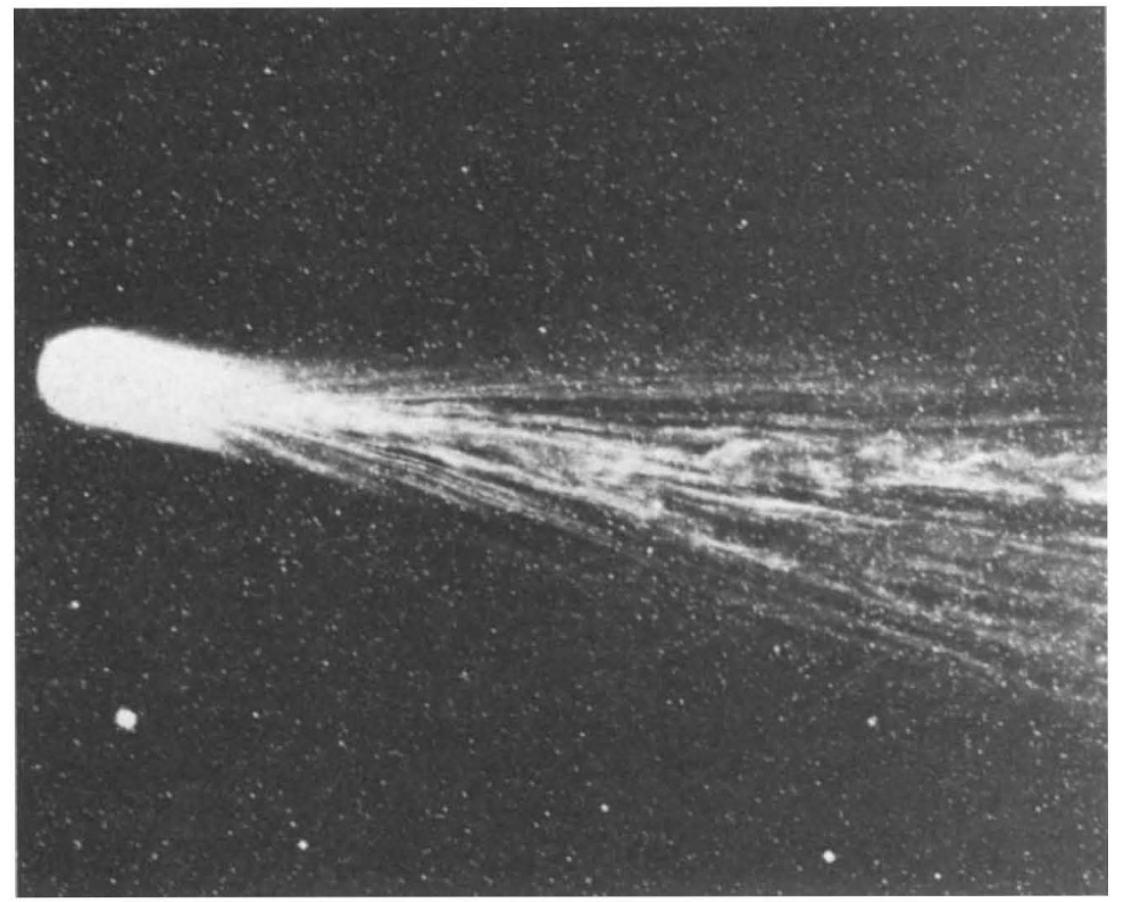

The delicate and complex details in the ion tail of comet Halley are revealed in this photograph taken on the 1.2-m UK Schmidt Telescope at Coonabarabran, New South Wales, Australia. The original 14-inch square plate has been subjected to special image enhancement techniques by David Malin of the Anglo-Australian Observatory to reveal this amount of detail which is normally hidden in the bright shroud of the dust tail. The structures seen here are produced by the interplay of the Sun's radiation and magnetic field on molecules released from the tiny cometary nucleus. The comet was at a distance of about 150 million kilometres when this 15-minute exposure was made. The bright fuzzy object near the head of the comet is the globular cluster Messier 75 at a distance of about 100,000 light years. First results from the Soviet, Japanese and European spacecraft encounters with comet Halley will be published in Nature on 15 May. 